\title{
EVOLUCIÓN DE LA VEGETACIÓN EN LOS ÚLTIMOS 6000 AÑOS EN LOS SECTORES CENTRAL Y ORIENTAL DEL SISTEMA CENTRAL ESPAÑOL
}

\author{
Blanca RUIZ ZAPATA ${ }^{1}$, Antonia ANDRADE OLALLA ${ }^{1}$, María J. GIL \\ GARCÍA ${ }^{l}$, Miriam DORADO VALIÑO ${ }^{1}$ y Miguel ATIENZA BALLANO ${ }^{1}$ \\ ' Departamento de Geología. Facultad de Ciencias. Universidad de Alcalá. 28871 Alcalá de Henares. Madrid.
}

\begin{abstract}
Ruíz Zapata, B., Andrade Olalla, A., Gil García, M.J., Dorado Valiño, M. y Atienza Ballano, M. 1996. Evolución de la vegetación en los últimos 6.000 años en los sectores central y oriental del Sistema Central español. [Evolution of the vegetation during the last 6.000 years in the eastern and central sectors of the Spanish Central System]. Revista Española de Paleontología, Nº Extraordinario, 288-298. ISSN 0213-6937.
\end{abstract}

\begin{abstract}
The palynologic results from five peats localized in the central and oriental sectors of the Spanish Central System are presented in order to reconstruct the vegetation evolution in the area and the paleoclimatic implications, due to pollinic content of the peat material. The beginning of this evolution is situated earlier than 6,000 years B.P. until the present time, due to ${ }^{14} \mathrm{C}$ datations. The anthropic factor is the most important cause of the vegetation development during, at least, the last 3.000 years.
\end{abstract}

Keywords: Pollen, Peats, Paleoclimate, Anthropic action, Holocene, Spanish Central System.

\section{RESUMEN}

Se presentan los datos de carácter palinológico correspondientes a cinco turberas localizadas en los sectores central y oriental del Sistema Central Español. Los objetivos de nuestro trabajo es poder reconstruir, en base al contenido en polen de dicho material, la evolución de la vegetación en el área y sus implicaciones de carácter paleoclimático. Las dataciones de ${ }^{14} \mathrm{C}$ sitúan el inicio de esta evolución con anterioridad a los 6.000 años B.P. hasta la actualidad. El factor antrópico es la causa más importante en el desarrollo de la vegetación durante, al menos, los últimos 3.000 años.

Palabras clave: Polen, Turberas, Paleoclima, Acción antrópica, Holoceno, Sistema Central.

\section{INTRODUCCIÓN}

Los objetivos fundamentales de este trabajo son, por una parte, reconstruir la evolución de la vegetación y del clima del Sistema Central Español durante el Holoceno y, por otra, determinar el grado de influencia de la actividad antrópica en el desarrollo de la vegetación. Para ello se presentan los datos de carácter palinológico que corresponden a una selección de 5 turberas, de las 36 analizadas, ubicadas en cada uno de los sectores central y oriental del Sistema Central Español.

El Sistema Central Español conforma una alineación montañosa a lo largo de unos $400 \mathrm{~km}$, de dirección general aproximada NE-SO. Se puede dividir en una serie de unidades montañosas independientes, que están constituídas por conjuntos geográficos menores, agrupados bajo el término de sierras, según Acaso (1983), delimitadas entre sí por Puertos o corredores que hacen que el Sistema Central no presente continuidad altimétrica. Dichos elementos pueden agruparse en tres grandes sectores (Fig. 1): el sector oriental, que posee una estructura sencilla, consta de una cadena montañosa principal, con Somosierra (2.250 m) y Guadarrama (2.406 m) y algunas ramificaciones secundarias, como la Sierra de Ayllón $(1.691 \mathrm{~m})$. El sector central, constituído por Gredos (2.592 m) y conjuntos menores, tales como La Paramera-La
Serrota (2.294 m), Villafranca-Piedrahita (2.095 m) y el sector occidental, constituído por Béjar $(2.401 \mathrm{~m})$ y Peña de Francia $(1.723 \mathrm{~m})$. Estas sierras, hasta cierto punto independientes y con dirección Este-Oeste, configuran un diseño en "H tendida", que presenta toda la cadena, tal como lo define Pedraza (1976). En todas ellas han quedado patentes las huellas de la acción modeladora de los procesos geomorfológicos cuaternarios, tales como los generados por fenómenos glaciares, fluviales, periglaciares y de ladera. Por ello se ha podido contar con la existencia de depósitos de turba, de origen intramorrénico, fluviolacustre y periglaciar, que han permitido la realización de este trabajo, el cual es una síntesis de la labor de investigación realizada durante los últimos 10 años por el Laboratorio de Palinología de la U.A.H.

El conocido carácter mediterráneo del Sistema Central no es debido a la escasa pluviosidad, ya que en muchas sierras es del orden de los $1.000 \mathrm{~mm}$, sino a la distribución de las precipitaciones. Estas se concentran en otoño y primavera y dependen de la dirección y frecuencia de los frentes de lluvia, los cuales están condicionados por la dirección de los vientos ábregos de componente NE y SO; ello trae consigo una mayor precipitación en los extremos del Sistema Central, quedando el sector Central sometido a una mayor continentalidad. Pese a ello existen numerosas variaciones locales, 


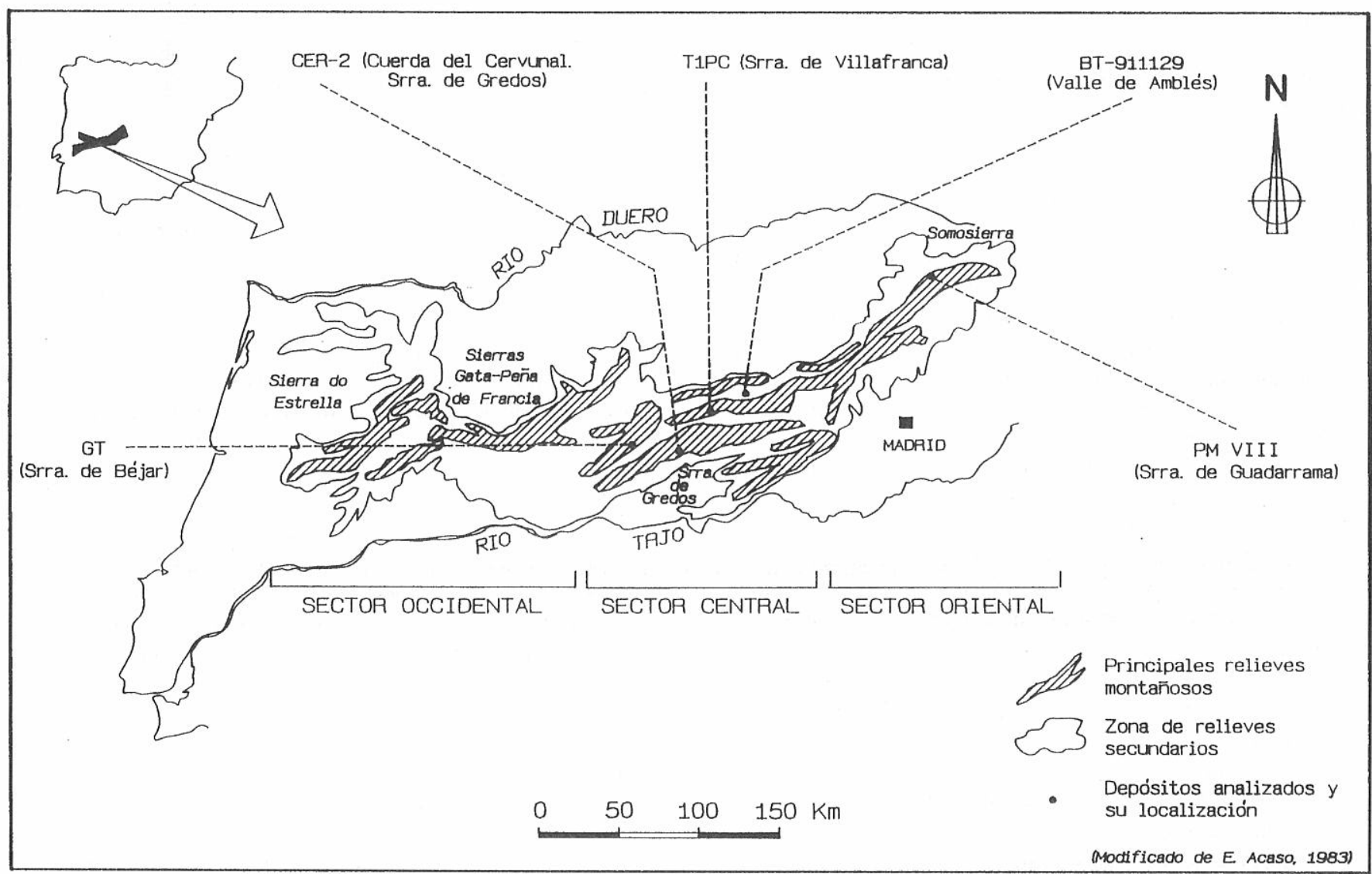

Figura 1. Situación geográfica y localización de los depósitos.

entre las que cabe destacar el efecto de sombra de lluvia producido por los altos macizos, como es el caso de Gredos, que ejerce un efecto pantalla sobre las sierras de Villafranca y La Paramera.

Tanto los parámetros climáticos, como la irregular topografía de todo el Sistema Central, hacen difícil resumir en este trabajo la vegetación característica de estas sierras. En este sentido, Rivas-Martínez (1975) considera los pisos de vegetación de la sierra de Gredos como ejemplo de cliserie de vegetación para el Sistema Central. Así, se puede hablar, en líneas generales, de la existencia del piso mesomediterráneo, representado fundamentalmente por el encinar y sus etapas de sustitución, que se sitúan en una banda de 350 a 800 $\mathrm{m}$ de altitud. El piso supramediterráneo está constituído principalmente por la serie de vegetación del melojar y sus etapas degradativas y se extiende entre los 800 y los $1.700 \mathrm{~m}$ de altitud, por término medio. El piso oromediterráneo posee una vegetación dominada por el piorno serrano y abarca desde los 1.600 a $2.100 \mathrm{~m}$. Finalmente, el piso crioromediterráneo, que se extiende hasta las cumbres más elevadas, presenta la serie de vegetación correspondiente al prado psicroxerófilo, aunque con frecuencia se halla ausente, debido a la topografía de las altas cumbres.

La oscilación de los límites de los pisos de vegetación depende de la exposición y el clima locales; además, junto a la cubierta vegetal dominante en un piso, existen en él otras formaciones menores, ligadas a los microambientes donde prosperan.

\section{MATERIAL Y MÉTODOS}

Desde el punto de vista litológico, todos los depósitos están constituídos por un material detrítico, más o menos grosero en la base, que progresivamente se va enriqueciendo en turba. Esta litología, unida al carácter de alta montaña de los depósitos, obliga al uso de sondas manuales (Hiller modificada y/o rusa), para la extracción del testigo.

Respecto al tratamiento químico de las muestras, se han empleado los protocolos propuestos, en base a la naturaleza litológica, es decir, ataque químico, mediante ácidos y álcalis $\mathrm{y}$, en algunos casos, se han tratado además, con líquidos densos para favorecer la concentración del polen en los casos en los que fue necesario. Los datos se han tratado estadísticamente con el programa TILIA (Grimm, 1992), para la realización de los Diagramas polínicos que presentamos.

\section{RESULTADOS}

A la vista de los objetivos propuestos, presentamos los Diagramas Polínicos más representativos tanto por su ubicación en los sectores central y oriental del Sistema Central, como por los resultados obtenidos. Dichos Diagramas Polínicos corresponden a los depósitos de la Sierra de Guadarrama, Valle de Amblés, Sierra de Villafranca, Gredos y Sierra de Béjar. 


\section{Gtrampal}

Se trata de un depósito de turba, desarrollado en un antiguo lecho de sobreexcavación glaciar, a una altitud de 1.440 $\mathrm{m}$, en la zona denominada La Garganta del Trampal, en la vertiente Este de la Sierra de Candelario (Avila), o mejor conocida como Sierra de Béjar: está situada en el horizonte superior de un melojar supramediterráneo.

La vegetación actual está formada principalmente por piornales de sustitución del melojar, vegetación de ribera, a lo largo de los abundantes arroyos que recorren la garganta y algunos cervunales instalados en áreas que cuentan con cierta humedad edáfica. En cuanto a la vegetación arbórea sólo quedan pequeñas manchas de abedulares, además de acebos y serbales aislados.

La superficie de la turbera no es homogénea, dado que posteriormente al fenómeno glaciar se ha instalado un caudaloso arroyo, el cual ha roto la antigua barrera rocosa que formó la laguna. Esto hace que esta pequeña cuenca esté en proceso de vaciado, por lo que algunas zonas muestran haber sido ya parcialmente erosionadas de sus sedimentos más superficiales.

Dentro de la propia turbera existen varios cauces encajados que la recorren, lo cual fué aprovechado para la toma directa del material sin necesidad de sonda.

En esta trinchera, denominada GTRAMPAL, se alcanzó una profundidad de $205 \mathrm{~cm}$, que tomados cada cinco centímetros, constituyen 41 muestras.

Litológicamente se observan dos zonas bien diferenciadas; una superior netamente orgánica hasta $120 \mathrm{~cm}$, y otra inferior con sedimentos progresivamente más detríticos hasta llegar a $170 \mathrm{~cm}$; a partir de aquí se suceden arenas pardas, arenas muy claras a $185 \mathrm{~cm}$ y los últimos $10 \mathrm{~cm}$ corresponden a arenas gruesas.

Desde el punto de vista polínico, salvo la muestra más profunda que resultó estéril, se han identificado 56 tipos polínicos en 12.803 granos de polen.

La riqueza polínica del sedimento es bastante variable, siendo muy elevada en las muestras de turba (muy orgánica) a nivel superficial (a $30 \mathrm{~cm} 126.133$ granos/gramo de muestra), y muy reducida en las muestras mas detríticas del fondo (646 granos/gramo de muestra a $200 \mathrm{~cm}$ ).

Se ha realizado una datación por Carbono-14 que a los 110-115 cm arroja una edad de 5.270 \pm 90 B.P.

Al observar el Diagrama Polínico (Fig. 2) se aprecia como nota más característica un dominio mayoritario de la vegetación arbórea, debido fundamentalmente a Betula, con la excepción de la parte central del diagrama y los niveles más superficiales en los que aparece en baja proporción.

El Diagrama se ha dividido en siete niveles en función de las fases de vegetación que aparecen.

Nivel G.1: De $200 \mathrm{~cm}$ a $185 \mathrm{~cm}$. Es el nivel inferior; queda caracterizado por un dominio y expansión de Betula, el cual hace que la proporción de polen arbóreo sea mayoritaria. Tambien aparece Pinus y Juniperus. Poaceae es el elemento más importante entre las herbáceas. Le siguen Apiaceae, Artemisia, Asteroideae y Cichorioideae.

Nivel G.2: De $185 \mathrm{~cm}$ a $135 \mathrm{~cm}$. Está definido por la estabilización del incremento de Betula $(70 \%)$; su descenso, coincidente con el inicio de la curva de Alnus, marca el final de este nivel. En esta fase continuan existiendo porcentajes significa- tivos de Pinus y Juniperus, así como una presencia muy puntual de Carpinus. La vegetación herbácea muestra aquí las proporciones mas bajas de todo el diagrama. Está constituida principalmente por Poaceae, Artemisia y Asteroideae.

Nivel G.3: En este nivel se sigue observando un dominio de la vegetación por parte de Betula. De $135 \mathrm{~cm}$ a 100 $\mathrm{cm}$. Sin embargo, está en claro descenso, ya que pasa de una presencia del $70 \%$ al $26 \%$. Continúan las mismas proporciones de Pinus y Juniperus, y las curvas de Quercus robur-t y Quercus ilex- $t$ pasan de ser meras presencias a tener cierta continuidad. Se da un desarrollo importante de las curvas de Poaceae y de Asteroideae por parte de las herbáceas. También aparecen cantidades destacables de Caryophyllaceae, Apiaceae y Fabaceae. En este nivel se sitúa la datación realizada por el método C-14, que arroja una edad de $5.270 \pm 90$ B.P., lo que permite definir una de las fases vegetales del periodo Atlántico en la Sierra de Béjar.

Nivel G.4 :De $100 \mathrm{~cm}$ a $60 \mathrm{~cm}$. En este tramo del perfil se aprecia una importante recuperación de la vegetación arbórea, primero por parte de Pinus, Quercus robur-t y Quercus ilex- $t$, quienes registran sus máximos en este tramo. Sin embargo sus proporciones se van a ver rebasadas en la parte superior de este nivel por una nueva y masiva extensión de Betula, quien alcanza de nuevo cifras del $65 \%$. En cuanto a las herbáceas, sólo Poaceae muestra una cantidad significativa al experimentar un cierto desarrollo en este nivel. Le siguen muy de lejos Fabaceae, Caryophyllaceae, Asteroideae y Cichorioideae.

Nivel G.5: De $60 \mathrm{~cm}$ a $30 \mathrm{~cm}$. Este nivel corresponde al intervalo en el que se aprecia un avance de la vegetación no arbórea, hasta el inicio de la curva continua de Castanea, a $30 \mathrm{~cm}$ de la superficie. Betula, que es quien caracteriza prácticamente la dinámica de la vegetación arbórea, registra un fuerte descenso, salvo al final con una expansión corta en el tiempo, pero intensa en valores. El registro de Pinus continua idéntico al del nivel anterior, al contrario que ambos tipos de Quercus, que muestran un sensible descenso. En este tramo aparece una curva casi continua de Fraxinus y la misma proporción de Juniperus que en tramos anteriores. La vegetación herbácea experimenta un pequeño avance, no tanto por parte de Poaceae, que mantiene niveles anteriores, como por parte de Asteroideae y Caryophyllaceae que experimentan un gran desarrollo. Ericaceae y Plantago inician su curva continua.

Nivel G.6: De $30 \mathrm{~cm}$ a $10 \mathrm{~cm}$. Se trata de una fase de retroceso continuo y definitivo de la vegetación arbórea hasta el valor del $20 \%$, caracterizada sobre todo por Betula y Pinus. Los dos tipos de Quercus mantienen sus proporciones y Castanea presenta una curva continua. En general se mantienen los porcentajes anteriores de todas las herbáceas, salvo Caryophyllaceae que reduce su representación y Poaceae que experimenta una fuerte expansión.

Nivel G.7: De $10 \mathrm{~cm}$ a $0 \mathrm{~cm}$. Los datos polínicos que presentan las dos últimas muestras son los que más claramente reflejan el impacto antrópico en el paisaje vegetal. Al mantenimiento de los bajos porcentajes de polen arbóreo y la continuidad de las curvas de Castanea y Olea, hay que añadir los altos valores alcanzados por Poaceae y Rumex, así como la presencia significativa de Ericaceae, Fabaceae, Plantago y sobre todo Cerealia.

En conjunto este diagrama se presenta totalmente definido por las curvas de Betula y Poaceae. Esto representa cierto riesgo a la hora de establecer las zonas, al ser estos unos 


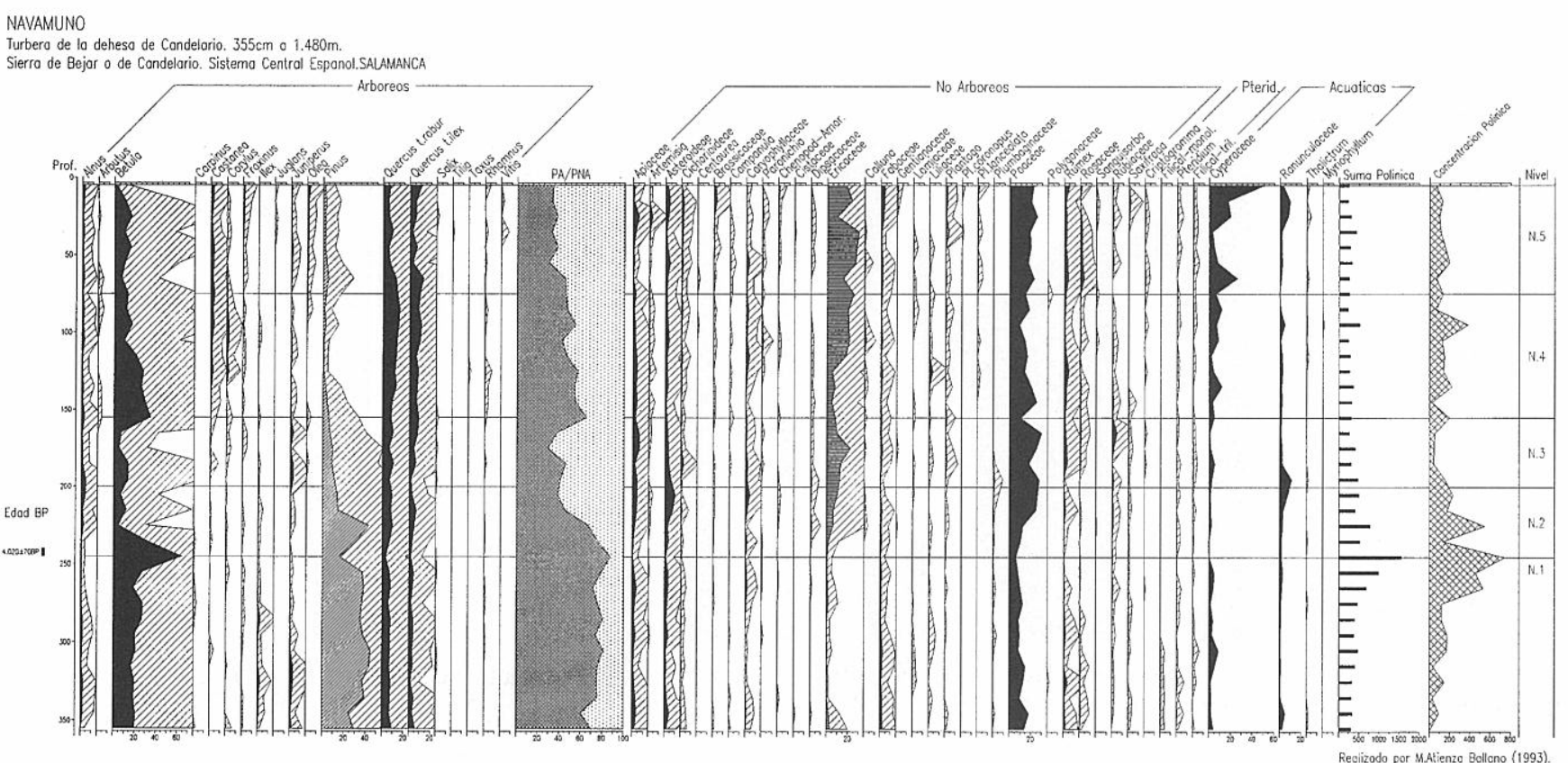

Figura 2. Diagrama polínico Sierra de Candeleda (GTRAMPAL).

elementos con marcado carácter en cuanto a la lluvia polínica local.

Lo anteriormente expuesto se ha tenido en cuenta, así como otras indicaciones extraidas del estudio de la lluvia polínica actual, en la definición de las zonas polínicas, en base a la dinámica de otros tipos polínicos de mayor expresión, dentro de la lluvia polínica regional, como es el caso de Pinus, Ericaceae, Quercus robur-t, Castanea y Olea (Atienza, 1993).

\section{Cer-2}

El Diagrama Polínico CER-2 (Fig.3), corresponde a un depósito situado en la cuerda del Cervunal a $1.840 \mathrm{~m}$ de altitud. Se trata de un depósito de carácter intramorrénico, generado por las morrenas laterales de los glaciares de Gredos y de el Pinar (Ávila). El depósito, con una profundidad de 3 metros, está constituído por sedimentos finos, con alto contenido en materia orgánica, sobre el que se desarrolla un suelo de césped alpino (Festuca indigesta).

A la vista de los datos reflejados en el diagrama polínico, este se ha dividido en dos zonas, en base al desarrollo del la curva PA/PNA, es decir de la relación entre el componente arbóreo y no arbóreo; así,

Zona I, definida por valores de PA superiores al 50\%, debido fundamentalmente al desarrollo de Betula y presencia continua de Pinus, Salix y Corylus. Dentro de ella se han diferenciado dos subzonas dado el comportamiento de los taxa principales; de este modo hablamos de:

Ia. retroceso de Pinus, aumento de Betula y presencia de Alnus y Quercus. Se detectan presencias de Asteraceae, Chenopodiaceae y Poaceae.

Ib. máximo de Betula, retroceso acusado de Pinus y desaparición de Alnus y Quercus. La vegetación no arbórea, está domimnada por elementos acuáticos, fundamentalmente Cyperaceae. Tambien cabe destacar a Ericaceae y Poaceae.

Zona II, con un marcado retroceso del estrato arbóreo. Esta división se ve corroborada por los valores que alcanzan los componentes principales, así como la mayor variación dentro del estrato no arbóreo; por ello y como en el caso anterior se ha subdividido en:

IIa. caracterizada por el desarrollo de Pinus, el cual sustituye al abedul y el inicio de la curva de Quercus. Junto a ellos, cabe destacar la presencia de Alnus y la caída de Corylus. Se detectan presencias de Asteraceae, Chenopodiacaceae, y una mayor variedad de elementos acuáticos.

IIb. establecida en base al desarrollo de Quercus, así como el de Salix, caída de Pinus, pese al aumento que sufre ya en las muestras más superiores, presencias esporádicas de Alnus y Corylus y práctica desaparición de Betula.Tambien destaca el aumento experimentado por Poaceae.

Con todo ello puede apreciarse, que desde el punto de vista cualitativo, el diagrama polínico guarda una relativa homogeneidad. El desarrollo de la vegetación no arbórea se ve enmascarada por la presencia de elementos acuáticos, de carácter totalmente local, reflejando la estabilidad de las condiciones locales, durante la formación del depósito. Al no contar con dataciones absolutas para este depósito, su ubicación en el tiempo se ha realizado en base a la similitud de los datos con los procedentes de otros depósitos de la zona. Este diagrama recoge tres fases de vegetación, que a nivel regional las hemos ido reconociendo en otros puntos del Sistema Central y que se resumen en una primera fase de dominio del abedul (Ia y Ib), que determina unas condiciones de humedad ambiental, mayores que las reinantes en la actualidad y que a nivel local se manifiesta en el gran desarrollo de elementos acuáticos. Una segunda fase caracterizada por el dominio del 


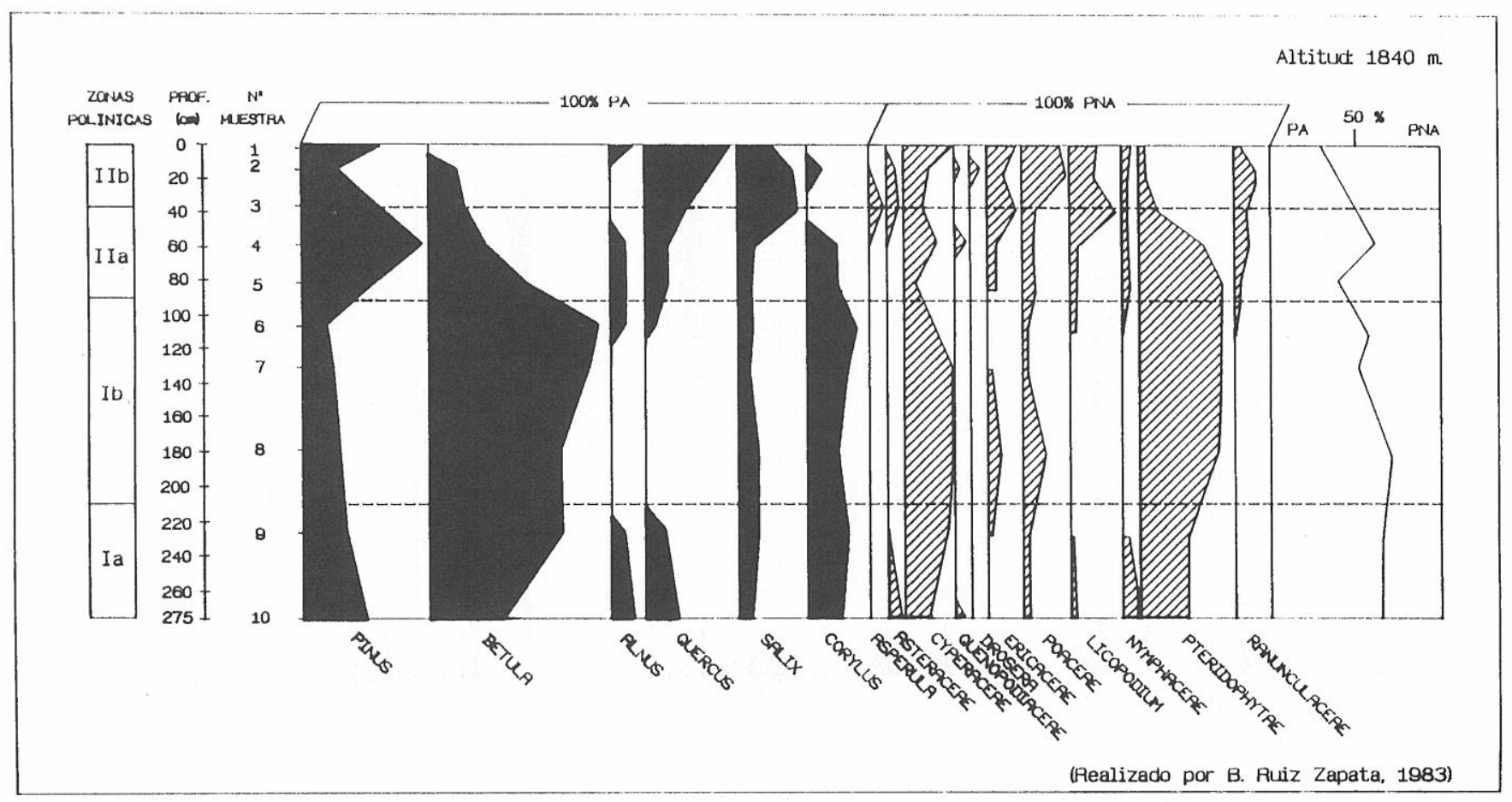

Figura 3. Diagrama polínico Sierra de Gredos (CER-2).

pino (IIa) y una tercera fase de dominio de Quercus (IIb), es decir el tránsito desde unos bosques de tipo atlántico a bosques de tipo mediterráneo. La acción antrópica queda reflejada por el descenso del componente arbóreo, sin embargo no existen claros indicios para hablar de una intervención de tipo agrícola y/o ganadera y solo un hecho, relativamente reciente, deja su huella, en términos polínicos: la repoblación del área, como lo pone de manifiesto el aumento de la curva del pino, en las muestras superiores (Ruiz y Acaso, 1981).

\section{T1PC}

El sondeo realizado en el Puerto de Chía, en la Sierra de Villafranca, se halla a una altitud de $1.701 \mathrm{~m}$. T1PC, tiene $200 \mathrm{~cm}$ y litológicamente, consiste en material detrítico fino con gran cantidad de materia orgánica, la cual disminuye hacia la base de la trinchera, apareciendo "lem" de color ocre.

Tenemos que decir que, a la vista de la trinchera y del sondeo "in situ", geológicamente se comporta como una "terraza fluvial", de forma que se originó por la erosión del cauce fluvial que cruza a través del Puerto de Chía (Arroyo del Puerto de Chía).

En cuanto al número de tipos polínicos que aparece en el Diagrama Polínico (Fig. 4), hay hasta 42 taxa que se distribuyen muy irregularmente a lo largo de todas las muestras: desde 8 hasta 24 y la media está en 15-16 taxa.

Basándonos en la evolución de las curvas de Polen Arbóreo/Polen no Arbóreo (PA/PNA) y las de los taxa encontrados, diferenciamos, en el total del registro analizado 5 zonas.

Zona I: Se define desde la base del T1PC (muestra 17), hasta la muestra 14. Se caracteriza por un desarrollo de la curva de PA, siendo Pinus el responsable de este máximo. Tambien contribuyen, pero en pequeña medida, Quercus caducifolio y Salix, y del resto de tipos arbóreos, hay mínimas presencias. Los taxa no arbóreos tienen a Poaceae como elemento más importante y representativo y el resto, aunque ya comienzan con curvas continuas, no sobrepasan el $10 \%$.

Zona II: En la trinchera T1PC, desde la muestra 14 hasta la muestra 5, en esta zona se da el fenómeno contrario al de la zona anterior. La curva PA/PNA tiene un mínimo en torno a las muestras 8 y 9 (de un 20\%), cuyo principal responsable es Pinus, acusado tambien por Quercus caducifolio y el resto de tipos arbóreos sólo muestran pequeñas presencias en esta zona (Alnus, Betula, Oleaceae, Salix). Respecto a los tipos herbáceos, Poaceae aumenta hasta un $60 \%$ y aumentan taxa como Compositae liguli y tubuliflorae, y se mantiene la curva de Ericaceae, mientras que la de Caryophyllaceae disminuye en esta zona. Respecto a taxa antrópicos, Cerealia presenta aquí un aumento que coincide con el mínimo de Pinus, aunque este porcentaje no alcance más allá del $10 \%$. Además, hemos subdividido esta zona en dos subzonas, II a y II b, en base al mínimo de la curva PA/PNA, anteriormente comentado, en la muestra 8.

Zona III: Desde la muestra 5 hasta la muestra 3, en la trinchera T1PC; la zona III se caracteriza por un nuevo aumento del Polen Arbóreo, sobrepasando en la muestra 4 de T1PC el $80 \%$. Nuevamente, Pinus es el encargado de este aumento, acompañado por Quercus caducifolio durante todo su aumento, excepto en el momento de máximo porcentaje, durante el cual casi desaparece, para volver a incrementarse en la siguiente zona. Los tipos no arbóreos disminuyen sensiblemente respecto a la zona anterior, sobre todo los que fueron comentados para la zona II. Incluso la presencia del taxón 


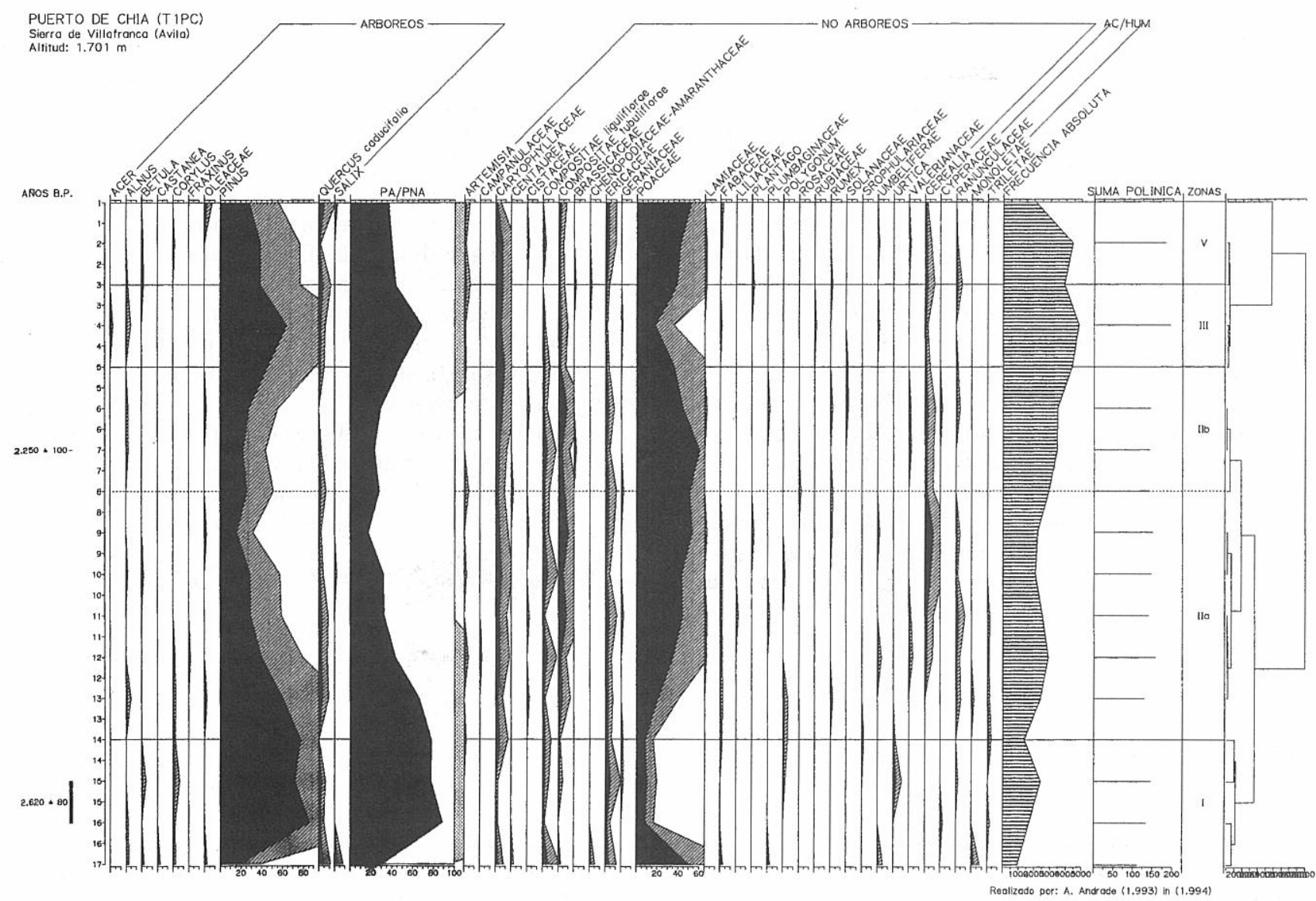

Figura 4. Diagrama polínico Sierra de Villafranca (T1PC).

antrópico Cerealia queda cortada en esta zona y no vuelve a aparecer hasta más adelante, así como la presencia de Polygonum y de Fabaceae; sin embargo, otros taxa antrópicos como Chenopodiaceae-Amaranthaceae y Rumex no aparecen en este intervalo, más que hacia el techo. Ranunculaceae presenta una curva a esta profundidad que solo se volverá a repetir hacia la superficie. El principal componente de los taxa herbáceos, Poaceae, tambien disminuye en esta zona y alcanza aquí su más bajo porcentaje.

Zona IV: Definimos esta zona como el nivel superior, cuyo significado es el de ser el suelo desarrollado en la parte superior de la trinchera, al igual que en cualquier terraza fluvial "típica". El comportamiento general es el de establecerse una estabilización definitiva de las curvas de todos los taxa, manteniendo ligeras tendencias, tanto al aumento como a la disminución, en porcentajes, según el caso.

Tambien hay que resaltar que la curva de frecuencia absoluta (o lo que es lo mismo, de riqueza polínica), disminuye de forma significativa, lo que pueda ser debido a las características intrínsecas de un suelo, es decir, a que un suelo es, en líneas generales, un producto de alteración de un sustrato, influído por las condiciones climáticas (lluvia, nieve, sequía, etc.), que además es aprovechado por la vegetación (que con sus raíces, atraviesan dicho suelo), y que, por tanto, todo ello puede hacer que disminuya este contenido polínico (Andrade, 1994).

\section{Baterna}

El sondeo BT-911129 corresponde a un depósito de origen fluvio-lacustre de orientación N localizado en el Valle de Amblés, a $1.180 \mathrm{~m}$ de altitud. La serie de vegetación a la que pertenece la zona es Querco pyrenaicae-Fraxineto angustifoliae $S$. y la vegetación actual es un prado húmedo.

El desarrollo de la vegetación permite dividir el Diagrama Polínico (Fig. 5) en 6 niveles o zonas polínicas:

Nivel I: Caracterizado por el dominio de un bosque caducifolio constituido por Betula y Quercus-c. Se pueden distinguir dos subniveles:

- Ia: Se define por la expansión de Betula junto con Salix y Alnus, mientras que Quercus-c y Pinus retroceden ligeramente. La vegetación no arbórea está representada por Poaceae, Asteraceae y Artemisia fundamentalmente.

- Ib: Caracterizado por el retroceso de Betula, Salix y Alnus mientras que Quercus-c ocupa el espacio dejado por los anteriores. Pinus se mantiene constante y Quercus-p se desarrolla. La vegetación no arbórea está estabilizada.

Nivel II: Este nivel refleja el retroceso progresivo del bosque húmedo, siendo sustituido por un bosque más xérico. Los porcentajes de PA son menores que en el nivel anterior y se observa una ligera tendencia al retroceso. Se diferencian dos subniveles: 
- IIa: Los taxa Betula y Quercus-c retroceden y su lugar es ocupado por Pinus. Los porcentajes de Quercus-p disminuyen ligeramente. La vegetación no arbórea está representada por los mismos elementos, destacando el desarrollo de Artemisia y Plantago así como una mayor presencia de Chenopodiaceae.

- IIb: Se observa una tendencia al retroceso del AP. Betula, Pinus y Quercus-p retroceden, mientras que Quercus-c y Salix aumentan ligeramente. La vegetación no arbórea se estabiliza. Artemisia retrocede y Plantago, Rubiaceae, Centaurea y Rumex se expanden.

Nivel III: Caracterizado por la recuperación del PA y por ser una etapa de transición entre el bosque húmedo y una vegetación más xérica. Se pueden distinguir dos subniveles.

- IIIa: Con una datación de $5.930 \pm 100$ BP (Beta57953), se caracteriza por un aumento de Quer- cus-c mientras que Pinus, Betula y Quercus-p están estabilizados. La vegetación no arbórea se caracteriza por la aparición de Cistaceae y la expansión de Poaceae, acompañada por Asteraceae-l y Caryophyllaceae.

- IIIb: Los porcentajes de PA disminuyen debido fundamentalmente al retroceso de Pinus y de Betula. Quercus-c y Quercus-p mantienen sus valores y Olea se desarrolla. La vegetación no arbórea está representada principalmente por Poaceae, Cistaceae, Asteraceae y Artemisia.

Nivel IV: Este nivel se caracteriza por la tendencia a la recuperación de PA, siendo Betula sustituido por Pinus. Se distinguen dos subniveles:

- IVa: La vegetación arbórea queda caracterizada por la estabilización de Pinus, Quercus-c, Quercus-p y Olea. La vegetación no arbórea está constituida por Poaceae, Asteraceae, Artemisia, Plantago y Rumex.

\section{BATERNA (AVILA)}

Altitud $1100 \mathrm{~m}$.

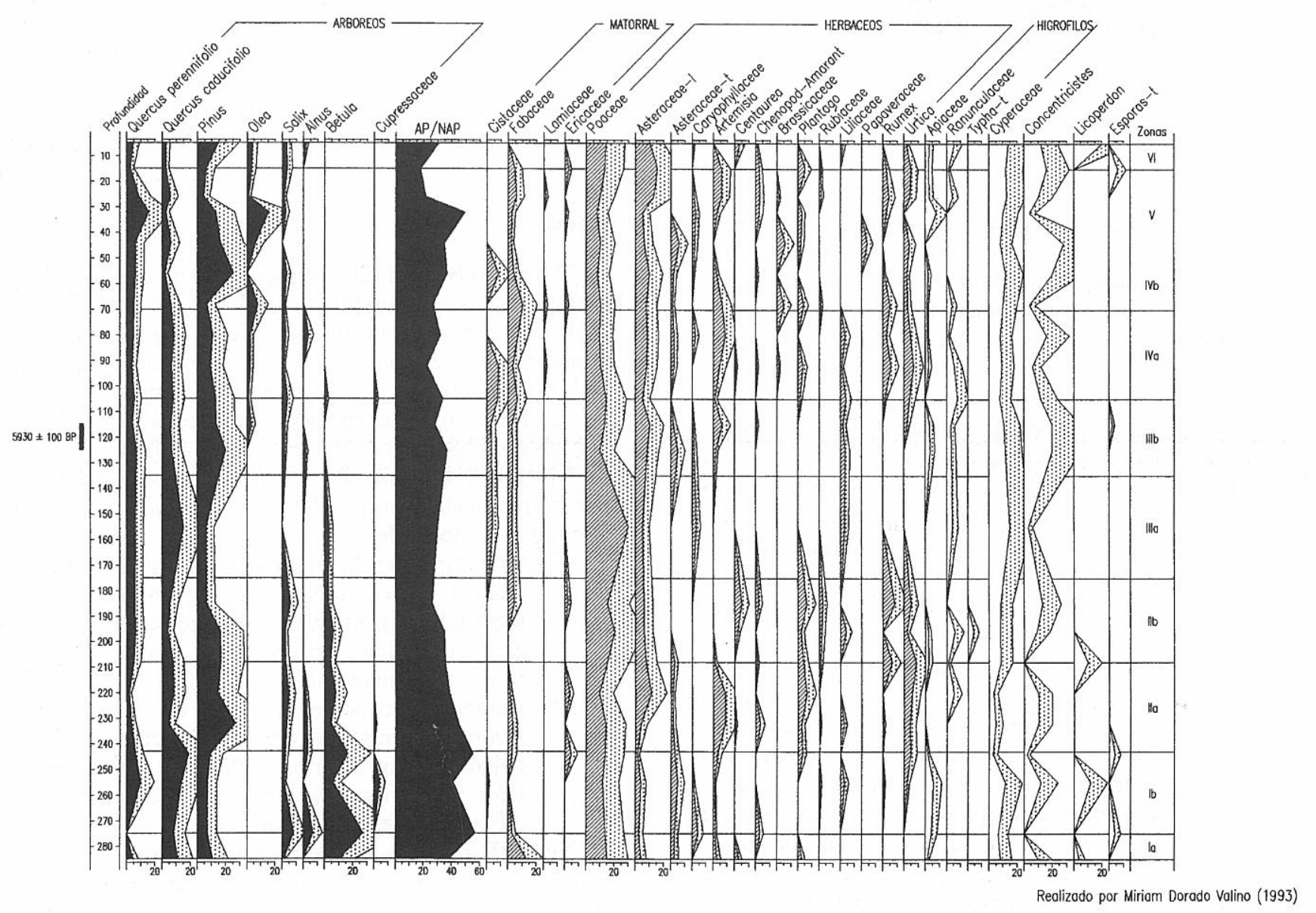

Figura 5. Diagrama polínico Valle de Amblés (BT-911129). 
- IVb: Con una datación de $2953 \pm 68$ BP, está caracterizado por la expansión del bosque de Pinus. Quercus-c y Olea también aumentan mientras que Quercus-c retrocede. La vegetación no arbórea disminuye, siendo Artemisia, Plantago y Rumex los taxa que retroceden.

Nivel V: Se define por el retroceso de la vegetación arbórea, caracterizado por la caída de Pinus, Quercus-p y Olea y la estabilización de Quercus-c. En cuanto a la vegetación no arbórea se observa un desarrollo de Poaceae, Asteraceae, Artemisia, Chenopodiaceae, Plantago y Rumex.

Nivel VI: Se observa la recuperación de AP debido únicamente al aumento de Pinus mientras que los demás taxa arbóreos están estabilizados. Poaceae aumenta pero Asteraceae, Artemisia, Chenopodiaceae, Plantago y Rumex retroceden (Dorado, 1993).

\section{PM VIII}

Se ha realizado un sondeo correspondiente a la turbera denominada PM VIII, localizada en el Puerto de la Morcuera, a 1.750 m de altitud, en la Sierra de Guadarrama. A continuación, vamos a realizar una descripción detallada de todo el perfil (Fig. 6) tomando como base las tres zonas polínicas establecidas, mediante la zonación automática del G1PAL, junto con su composición florística.

Zona a: abarca los espectros 28 a 34 comprendidos entre 140 a $195 \mathrm{~cm}$. En esta zona se aprecia un claro predominio del componente arbóreo, siendo Pinus el taxón dominante llegando a alcanzar el $81,4 \%$. De forma continua, pero con valores inferiores, se encuentran Quercus- c. у Betula, mientras que Corylus y Juniperus están presentes más esporádicamente. El componente herbáceo se encuentra representado fundamentalmente por Poaceae, Ericaceae y Asteroideae, y de forma dispersa se encuentran Caryophyllaceae, Rumex y Artemisia.

Zona b: en ella se incluyen los espectros 8 a 27 correspondientes al intervalo entre 40 a $140 \mathrm{~cm}$. Se ha subdividido en tres subzonas en base a su composición florística. Las subzonas b1 y b3 son muy similares en cuanto a su contenido florístico, si bien en la b3 se observa un claro retroceso de Pinus coincidente con un mayor desarrollo de Quercus- c. En ambas, Pinus es el taxón mejor representado seguido de Quercus-c., además se encuentran presentes de forma más esporádica y con menores frecuencias Betula, Acer, Corylus, Juniperus y Salix. Dentro del componente herbáceo, los taxones mejor representados son Poaceae, Asteroideae y Apiaceae, mientras que Ericaceae, Caryophyllaceae, Cichorioideae y Polygonum se encuentran mejor representados en la subzona b3.

La subzona b2, está caracterizada por un brusco descenso y posterior aumento del componente arbóreo, propiciado por Pinus mientras que Quercus-c sufre un ligero aumento. El resto de los taxones arbóreos tan sólo se encuentran puntualmente. En el estrato no arbóreo Poaceae, Asteroideae y Apiaceae se encuentran bien representadas, además se observa un incremento de Artemisia, Plantago y Rumex.

Zona c: constituida por los espectros 1 a 7 situados entre 0 y $40 \mathrm{~cm}$, se diferencia por una clara tendencia al retroceso por parte del componente arbóreo, descenso que es más brusco en el inicio de la zona. Este componente estaría repre- sentado por Pinus y Quercus c. principalmente. En los niveles inferiores se encuentran Alnus y Corylus, mientras que Juniperus y Salix lo están en los superiores. En cuanto a los taxones herbáceos se observa un rápido incremento de las frecuencias de Poaceae hacia los espectros superiores y ligeros aumento de Apiaceae, Asteroideae y Rumex, al tiempo que Artemisia, Cichorioideae y Ericaceae disminuyen hasta desaparecer (Gil, 1992).

\section{DISCUSIÓN}

Los datos polínicos que se presentan corresponden a 5 de los 36 depósitos más representativos de los sectores central y oriental del Sistema Central Español. Los Diagramas Polínicos presentados se seleccionaron debido a que, dentro de cada una de las zonas analizadas por cada uno de los autores, eran las que mejor representaban las características polínicas de cada zona y, juntas, podían reflejar la dinámica de la vegetación, tanto en el espacio, como en el tiempo. Las dataciones $\mathrm{C}^{14}$ (BETA ANALYTIC, UGRA) permiten conocer la evolución de la vegetación, y por tanto del clima, con anterioridad a los $5.930 \pm 100$ años B.P.

Pese a las características particulares de cada zona de estudio, que evidentemente imprimen un marcado carácter en la evolución de la vegetación, en conjunto, el área queda definida por la presencia de un componente arbóreo, constituído por Betula, Pinus y Quercus (Ruiz et al., 1992) y un conjunto, no arbóreo, dominado por Poaceae y en menor medida, por Asteraceae y Ericaceae. Junto a ellos, se detectan presencias, más o menos continuas de otros elementos, altamente significativos y cuyo desarrollo, en los distintos niveles de los diagramas polínicos, va ligado a determinadas condiciones ambientales y/o a los efectos de la acción antrópica en el entorno.

En base a las dataciones absolutas, tanto de los diagramas polínicos presentados como a las de otros depósitos del área y al desarrollo de las curvas de los componentes principales de la vegetación, podemos establecer una secuencia de fases de vegetación que desarrollamos a continuación.

Con anterioridad a los 6000 años B.P. (GTRAMPAL, Fig. 2; BT-911129, Fig. 5 y CER-2, Fig. 3), Betula domina en el área, si bien este dominio es más acusado en las sierras de Béjar y Gredos. Esta vegetación es reflejo por un lado del papel de Betula como colonizador de espacios abiertos; por otra parte su desarrollo y su permanencia, definen la existencia de bosques de tipo atlántico, lo que es indicador de un régimen de precipitaciones mucho mayor que el actual. Sin embargo, pese a esta tónica general, puede apreciarse la influencia del factor local, en cada uno de los depósitos estudiados; así, en la sierra de Béjar, la presencia de Betula es grande y constante en todo el perfil, mientras que en la sierra de Avila, al Norte del valle de Amblés (Dorado, 1993), situada más al este que Béjar y por tanto, con un mayor grado de continentalidad, su presencia supera con dificultad los 5930 \pm 100 años B.P. La sierra de Gredos, con una posición geográfica intermedia, muestra un desarrollo que podemos considerar de "tránsito" entre ambos; para estas edades no se cuentan con datos para el sector oriental del Sistema Central.

Asi pues, se aprecia una primera fase definida por el desarrollo de bosques de tipo atlántico, cuyo desarrollo espa- 


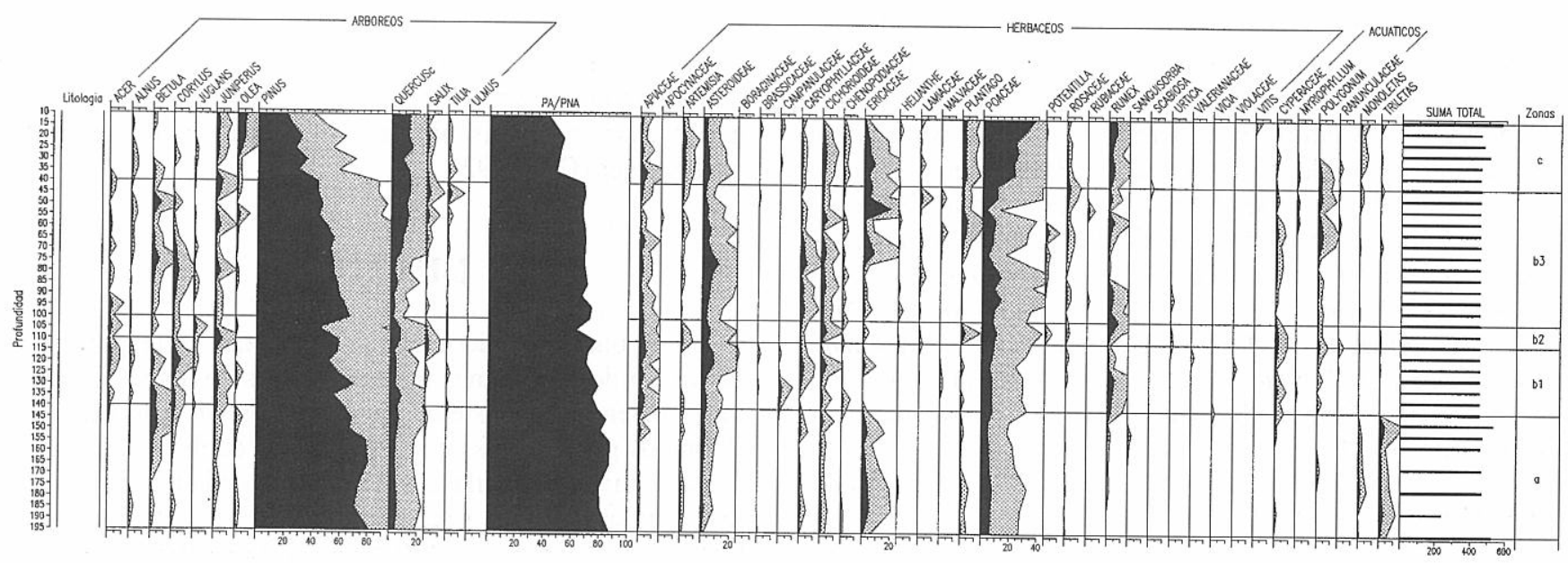

Realizodo por: M. Jose Gil Garcia (1994)

Figura 6. Diagrama polínico Sierra de Guadarrama (PMVIII).

cial y temporal queda condicionado por la mayor o menor continentalidad del área.

El protagonismo de Betula va a ser sustituído primero por Pinus y posteriormente por Quercus, es decir, que se aprecia una transición hacia un bosque de carácter mediterráneo. Este efecto se ve amortiguado en la sierra de Béjar y es muy patente en la de Gredos. Pese a que este fenómeno no es sincrónico (alrededor de los 5000 años B.P. en sierra de Béjar y de los 5900 B.P. en el valle de Amblés), debido posiblemente a la situación geográfica de cada depósito, sí permite apreciar la tendencia generalizada hacia unas condiciones de mayor sequedad. Esto se manifiesta igualmente en el descenso de los matorrales de Ericaceae y en el mayor desarrollo de Poaceae y Artemisia. Pese a que comienzan a detectarse presencias de taxa tales como Chenopodiaceae, Plantago y Rumex, las evidencias del impacto antrópico no son significativas. El retroceso del bosque y el dominio de Pinus queda patente alrededor de los $4020 \pm 70$ años B.P. (sierra de Béjar, Atienza, 1993) y en el valle de Amblés hacia los $2953 \pm 68$ B.P. (Dorado, 1993). Este mismo fenómeno, pese a no estar datado, se aprecia también en la sierra de Gredos (Ruiz y Acaso, 1983).

Dataciones más recientes permiten observar secuencias más detalladas de una nueva fase, que se detecta en todos los diagramas. Hacia los $2620 \pm 80$ años B.P. en el sondeo T1PC (Fig. 4) del Puerto de Chía (Andrade, 1994), se aprecia un último máximo de Pinus y presencias de Betula, quizá como consecuencia del aumento de pluviosidad, entre fases de grandes sequias, tal como Font Tullot (1988) define para el final del periodo Subboreal. Tras este episodio, Betula retrocede claramente, alli donde su presencia era aún abundante (sierra de Béjar) o desaparece, al tiempo que se detecta la presencia de Olea a nivel regional; este fenómeno en el valle de Amblés, donde su aparición fue temprana, queda de manifiesto por un máximo registrado en este taxa (Dorado, 1993).

Alrededor de los 2500 B.P. se aprecia el inicio de una intensa deforestación, asociada a la caida de Pinus de un modo gradual y a un mayor desarrollo de Quercus; este fenómeno puede detectarse en todo el área, e incluso a nivel peninsular, y es debida no solo al tránsito hacia unas condiciones más cálidas y secas, semejantes a las reinantes en la actualidad, sino que van reforzadas por el inicio de la actividad antrópica en el entorno; así, se aprecia la aparición o aumento de taxa ligados a ella, como son Olea, Plantago, Cerealia, Rumex, Artemisia, Chenopodiacaceae, como elementos más representativos. Evidentemente, esta deforestación tiene tambien unas peculiaridades locales muy marcadas, como en la sierra de Béjar (Atienza, 1993; Atienza, Dorado y Ruiz, 1991), donde se definen una primera fase ganadera, con predominio de Pinus, una segunda fase de cultivos de Castanea y Juglans y una tercera fase, tambien de cultivos, con Olea y Cerealia. En la sierra de Gredos, el fenómeno solo se detecta a nivel de deforestación y desarrollo de Quercus, pero no quedan claramente recogidos los taxa antrópicos, debido a la fuerte humedad local que enmascara los datos y la localización del depósito (Ruiz y Acaso, 1.981); algo similar ocurre en la sierra de Villafranca (Puerto de Chía), pese que en este diagrama se observan presencias puntuales de Olea, Cerealia y Artemisia; sin embargo, en ambos se detecta un aumento significativo de Poaceae (Andrade, 1994). En Baterna, en el valle de Amblés, existe una utilización temprana del territorio, con fines agrícolas, detectado por el desarrollo de Poaceae, Artemisia, Chenopodiaceae, Plantago, Rumex y Urtica (Dorado, 1993).

Finalmente, este mismo fenómeno queda registrado en el sector oriental, aunque en este caso se ve amortiguado por una mayor densidad de la cobertura vegetal arbórea, en comparación con los casos anteriores; así, el inicio de la deforestación queda patente con anterioridad a los $1710 \pm 90$ años B.P. (Gil, 1992). Pese a la densidad de la masa de Pinus, se observa, más que una sustitución, un aumento de Quercus junto al desarrollo de Poaceae, Olea, Juglans y Artemisia. Todo ello queda influenciado por unas características pecu- 
liares que presenta esta zona y que conviene resaltar, como son: la presencia de Betula, Corylus y Ericaceae, en porcentajes más significativos que en las otras áreas, debido a la influencia de los microclimas más húmedos desarrollados en los alrededores (sierra de Ayllón) (Gil, 1992 op. cit.), así como un mayor dominio del uso ganadero, sobre el agrícola, similar al observado en el Puerto de Chía (Andrade, 1994) y que sólo, para épocas muy recientes, se detectan Olea e incluso Vitis.

El desarrollo de esta vegetación queda explicada muchas veces por eventos de carácter histórico, así como la última expansión de Pinus, coincidente con la dominación romana y con el denominado "Periodo Cálido Romano". Este hecho queda patente claramente en el Puerto de Chía y en Morcuera; así como en el resto del territorio, el desarrollo de Cañadas Reales ya habla por sí solo del proceso de deforestacion generalizada. Esta regresión se acentúa en la época medieval y llega a afectar a los bosques de Betula en la sierra de Béjar y a los de Pinus en el resto del área, debida en gran parte al aumento de la ganadería, con la creación y desarrollo de la Mesta (fundada por Alfonso X El Sabio, en 1273). Esto provocó el descenso de la regeneración natural del bosque, no sólo por talas abusivas e incendios que provocaban los pastores, para la obtención de pastos, sino porque los brinzales de pino y otras arbóreas eran comidas sin discriminación alguna por el ganado.

El descenso del bosque llega a ser irrecuperable al menos hasta finales del siglo pasado y principios del actual, aunque se puede constatar la existencia de una conciencia sobre la conservación y repoblación de los bosques en muchos de los monarcas españoles (Rubio, 1989). Las medidas proteccionistas han permitido frenar este descenso e incluso provocar, bien por regeneración natural, bien por repoblación, nuevos aumentos en las superficies boscosas, los cuales se pueden evidenciar desde tres aspectos diferentes:

a. Por el aumento (debido a las últimas repoblaciones) en las concentraciones polínicas de aquellas árboreas que, de forma autóctona, dominaron el territorio, como por ejemplo, el caso de Pinus.

b. Presencia polínica de otros taxa que ante las nuevas condiciones (desapariciones de aquellos que les producían sombra) se han favorecido en su germinación, como es el caso de Quercus.

c. La aparición de nuevos taxa introducidos, como es por ejemplo el caso de Tilia, Corylus, Castanea y otros elementos, que han sido utilizados como ornamentales o para su explotación y que en la actualidad están naturalizados en ciertas áreas de nuestro Sistema Central.

\section{CONCLUSIONES}

A la vista de todo lo expuesto, podemos concluir que la evolución de la vegetación en el área de estudio durante los últimos 6000 años, queda definida por una serie de fases de carácter regional, detectables en todos los Diagramas Polínicos del área. Así podemos hablar:

- De la existencia de bosques de carácter atlántico con anterioridad a los 6000 B.P. y cuya distribución temporal queda definida por las características de mayor oceanidad o continentalidad en cada punto. Estas reflejan unas condiciones más húmedas y frescas que las actuales.

- Del paso gradual a bosques de carácter mediterráneo, como consecuencia de una transición a condiciones más cálidas o secas.

- Del inicio de la deforestación asociada a una más o menos intensa actividad antrópica en el entorno. Las modalidades que revisten dichas actividades se centran fundamentalmente en el uso ganadero, principalmente y/o agrícola, que se ponen de manifiesto por:

- El aumento de la presencia de taxa tales como Plantago, Polygonum, Rumex y Chenopodiaceae.

- Estar constatados por sucesos históricos: la creación y desarrollo de La Mesta, la cual fue determinante para el declive de los bosques en los sectores de estudio del Sistema Central Español.

- Que las posteriores medidas de protección y de repoblación de los bosques peninsulares en general, más numerosas a partir del siglo XVI, no fueron suficientes para parar la deforestación, que particularmente en nuestro área de estudio era ya irreversible.

Por ello podemos concluir que los factores determinantes de la evolución de la vegetación en los sectores central y oriental del Sistema Central Español para los últimos 6000 años han sido:

- Factores climáticos, para el intervalo 6000 - 2500 años B.P.

- Factores antrópicos, acentuados más o menos por los climáticos, para el periodo comprendido entre los 2500 años B.P. hasta la actualidad.

\section{BIBLIOGRAFÍA}

Acaso, E. 1983. "Estudio del Cuaternario en el Macizo Central de Gredos”. Tesis Doctoral. Universidad de Alcalá. 475 pp.

Andrade Olalla, A. 1994. "Dinámica de la vegetación durante los últimos 3.000 B.P., en las Sierras de La Paramera, La Serrota y Villafranca (Avila), a partir del análisis polínico". Tesis Doctoral. Universidad de Alcalá. 276 pp.

Atienza Ballano, M.; Dorado Valiño, M. y Ruíz Zapata, B. 1991. "La Palinología en el estudio de la acción antrópica. Aplicación a dos depósitos localizados en la Sierra de Béjar y Sierra de Avila". Actas de Gredos. Boletín Universitario 11: 31-38.

Atienza Ballano, M. 1993. "Evolución del paisaje vegetal en las Sierras de Béjar y Francia durante el Holoceno, a partir del análisis polínico". Tesis Doctoral. Universidad de Alcalá. 247 pp.

Dorado Valiño, M. 1993. "Evolución de la vegetación durante el Holoceno en el Valle de Amblés (Avila). Estudio palinológico". Tesis Doctoral. Universidad de Alcalá. 212 pp.

Font Tullot, I. 1988. "Historia del clima de España. Cambios climáticos y sus causas". Instituto Nacional de Meteorología. Madrid. 297 pp.

Gil Garcia, M. J. 1992. "Dinámica de la paleovegetación en el Sector Oriental del Sistema Central Español durante el Holoceno, en base al análisis polínico. Implicaciones climáticas". Tesis Doctoral. Universidad de Alcalá. 211 pp.

Grimm, E. C. 1992. "Tilia and Tilia-Graph: pollen spreadsheet and graphics programs". 8th International Palynological Congress:56. 
Pedraza, J. 1976. “Algunos procesos morfogenéticos recientes en el valle del rio Alberche (Sistema Central Español). La depresión de Aldea del Fresno-Almorax". Bol.Geol. y Min.,Tomo LXXXVII-1:1-12.

Rivas Martínez, S. 1975. "Mapa de vegetación de la provincia de Avila". Anal. Inst. Bot. Cavanilles, 32(2): 1.493-1.556.

Rubio Recio, J. M. 1989. "Biogeografía. Paisajes vegetales y vida animal”. Colección Geografía de España. Ed. Síntesis. 169 pp.

Ruiz Zapata, B. y Acaso Deltell, E. 1981. "Contribución al estudio del cuadro vegetal y climático durante el Cuaternario reciente en el Macizo Central de Gredos (Avila)". Bol.R.Soc.Esp.de Hist. Nat. (G),79:299-307
Ruiz Zapata, B. y Acaso Deltell, E. 1983. “Análisis polínico de un depósito lacustre en el Macizo Central de Gredos (Avila)". Actas del IV Simposio de Palinología: 423-432.

Ruiz, B.; Gil, M. J.; Andrade, A.; Atienza, M.; Dorado, M.; Gomez, A. y Martin T. 1992. "Evolución durante el Cuaternario Reciente de los taxa arbóreos más representativos en el Sistema Central y Sistema Ibérico". Actas del II Congreso Geológico de España y VII Latino-Americano. Tomo I: 554-559.

Manuscrito recibido: 5 de noviembre, 1994 Manuscrito aceptado: 14 de julio, 1995 